\title{
Propiedades psicométricas de los cuestionarios Reactive/Proactive Questionnaire (RPQ) y How I Think Questionaire (HIT) en estudiantes peruanos
}

\author{
María E. Rojas ${ }^{1}$, Walter L. Arias ${ }^{2}$, Renzo Rivera ${ }^{3}$, Jenny A. Geldres ${ }^{1}$, Marlene A. Starke ${ }^{1}$ \\ and Evert N. Apaza ${ }^{1}$ \\ ${ }^{1}$ Universidad Nacional de San Agustín, Arequipa, Perú \\ ${ }^{2}$ Universidad Continental, Arequipa, Perú \\ ${ }^{3}$ Universidad Católica San Pablo, Arequipa, Perú
}

Psychometric properties of the reactive/proactive questionnaire (RPQ) and How I Think Questionaire (HIT) in Peruvian high school students

\begin{abstract}
The present study aims to value the relationships between reactive/proactive aggression and cognitive distortion among adolescents from Arequipa City in Peru, in order to it was necessary to practice a psychometrical analysis of the tests used. In that sense there were evaluated 2830 high school students (48.9\% female and $51.1 \%$ male) aged 13 and 19 years old, with Reactive/Proactive Aggression Questionnaire and the How I Think Questionnaire. The psychometrical analysis included the estimation of the validity and reliability of the instruments applied, so as the correlation analysis to determine the relation between the variables. The psychometrical results shown that the construct validity and the reliability of the questionnaires, have good fit values and internal consistency; while the correlation analysis reveal that the reactive aggression is weakly related with cognitive distortions, and proactive aggression is related y moderate way cognitive distortions.
\end{abstract}

Key words: Reactive aggression; proactive aggression; cognitive distortion; psychometrics.

Resumen: La presente investigación tiene por finalidad valorar las relaciones entre la agresión reactiva/proactiva y las distorsiones cognitivas de adolescentes de la ciudad de Arequipa (Perú), para ello ha sido necesario realizar un análisis psicométrico de los instrumentos utilizados. En ese sentido se evaluó a 2830 estudiantes nivel secundario (48.9\% mujeres y 51.1\% varones) entre 13 y 19 años de edad, a través del Cuestionario de Agresión Reactiva/Proactiva y el Cuestionario How I Think. Se realizó un procesamiento psicométrico para valorar la validez y la confiabilidad de los instrumentos, así como un análisis de correlación para determinar el grado de relación entre las variables. Los resultados psicométricos dan cuenta de la validez de constructo y la confiabilidad de los instrumentos, que tienen índices adecuados de ajuste y consistencia interna; mientras que el análisis de correlación reveló que la agresión reactiva se relaciona de manera débil con las distorsiones cognitivas, y la agresión proactiva se relaciona de manera moderada con las distorsiones cognitivas.

Palabras clave: Agresión reactiva; agresión proactiva; distorsión cognitiva; psicometría.

\section{Introducción}

Recibido: 6 de marzo 2019; aceptado: 27 de marzo 2020.

Correspondencia: Walter L. Arias Gallegos. Universidad Continental, Av. Los Incas s/n, Arequipa, Perú. Correo-e: warias@continental.edu. pe
La agresión es una conducta adaptativa frente a una situación de amenaza, que se torna inadaptativa cuando se da de manera injustificada e irracional, y recibe el nombre de violencia. En ese sentido, los países de Amé- 
rica Latina registran índices de violencia muy elevados, siendo los tipos de violencia más recurrentes, la violencia intrafamiliar hacia las mujeres (64\%), hacia los niños (63\%), el acoso escolar (49\%), y la violencia de Estado (42\%); mientras el país más inseguro es Honduras el más seguro es Chile (Latinobarómetro, 2018).

La conducta agresiva obedece a diversos factores de tipo biológico y ambiental, pudiendo manifestarse desde la niñez. Biológicamente, hormonas como los andrógenos y la testosterona se asocian con la conducta agresiva, además de ciertas estructuras cerebrales como la amígdala y el lóbulo prefrontal, y neurotransmisores como el GABA, la serotonina, y la mono amino oxidasa (Gallardo-Pujol, Forero, Maydeu-Olivares y Andrés-Pueyo, 2009). Sin embargo, una gran diversidad de estudios ubica las causas de la conducta agresiva en el ambiente social y familiar. De este modo, varios estudios señalan que la conducta agresiva es engendrada en la familia cuando existen patrones de maltrato hacia los hijos (Quiroz, et al., 2007), violencia entre los padres (Rodríguez, 2003), y entornos comunales violentos o marginales, donde se cunde la pobreza y la delincuencia (Herrera y Morales, 2005).

La conducta agresiva es además importante, porque acarrea consecuencias negativas para los niños y adolescentes que las manifiestan. Así, la agresividad se ha asociado negativamente con la autoestima y el autoconcepto, y positivamente con la conducta antisocial (Garaigordobil, Durá y Pérez, 2005) y violencia hacia la pareja (Boira y Jodrá, 2010). También se señalan aspectos sociodemográficos como el sexo, que suelen reportar diferencias marcadas con una mayor predominancia de conductas agresivas y antisociales en los varones con respecto a las mujeres y aspectos culturales que actúan como factores diferenciales de la expresión de la agresividad (Torregrosa et al, 2010).

Por otro lado, existen diversas teorías sobre la agresividad e incluso diversas tipologías que intentan clasificar la conducta agresiva (Amor, Echeburúa y Loinaz, 2009); siendo una de ellas, la teoría de Dodge, que distingue entre agresión reactiva y proactiva (Dodge y Coie, 1987). La agresión reactiva describe conductas que responden a ciertas provocaciones o amenazas como la que ocurre por lo regular en los casos de violencia de pareja (Fernández y Echeburúa, 2008), y está basada en el modelo de frustración/agresión planteado por John Dollard (Dollard, Doob, Miller, Mowrer y Sears, 1939); mientras que la agresión proactiva, es un tipo de agresión que ocurre de manera deliberada y que comporta beneficios para el agresor, sobre la base de la teoría de aprendizaje social de Albert Bandura (1965), como la que acontece en casos de conductas delictivas o propiamente criminales (Andreu y Peña, 2013). Por ello, la agresión reactiva ha sido denominada como expresiva, emocional o caliente; mientras que la agresión proactiva ha sido denominada instrumental, razonada o fría (Andreu, 2010).

En ambos casos, empero, se han hecho estudios en Estados Unidos que relacionan estas formas de agresión con diversos síntomas y trastornos psicopatológicos. Así, la agresión reactiva se asocia con miedo, irritabilidad, hostilidad, sesgos en el procesamiento de la información, depresión y somatización (Dodge, Lochman, Harnish y Bates, 1997); mientras que la agresividad proactiva se ha relacionado con una valoración positiva de la agresión, ansiedad, impulsividad, distorsión de la realidad y personalidad psicopática (Raine et al., 2006).

Estudios realizados en adolescentes hispanoparlantes señalan que tanto la agresión reactiva, como la agresión proactiva, o su expresión mixta, se asocian con la conducta antisocial (Penado, Andreu y Peña, 2014). Asimismo, estudios en niños y adolescentes uruguayos han reportado que la agresión proactiva tiene a disminuir con la edad y que es más frecuente en varones (Fares, Cabrera, Lozano, Salas y Ramírez, 2012). Otros estudios han relacionado la agresividad reactiva, proactiva y mixta con variables cognitivas como la impulsividad cognitiva (Andreu, Peña y Penado, 2013), señalando que si bien los tres tipos de adolescentes agresivos muestran niveles elevados de impulsividad en comparación con sujetos no agresivos, los agresivos proactivos presentan mayores niveles de impulsividad no planificadora (Andreu, Peña y Penado, 2012). De este modo, el modelo de agresión reactiva/ proactiva o impulsiva/premeditada permite integrar dos perfiles diferenciales de conducta agresiva y antisocial, según diversos aspectos cognitivos y socioemocionales (Andreu, 2009).

Diversas teorías cognitivas como la de Beck y la de Ellis han puesto de manifiesto que las respuestas fisiológicas, afectivas y conductuales, dependen más que de los acontecimientos ocurridos, de las interpretaciones que hacemos de ellos, destacando así, la relevancia de las cogniciones en las regularidades y desviaciones de la conducta humana. De hecho, una variable cognitiva relacionada directamente con la conducta agresiva la constituyen las distorsiones cognitivas, de modo que los déficits en el procesamiento cognitivo de la información social se relacionan con patrones diferenciados de conducta agresiva: una atribución hostil sería propia de la agresión reactiva, y una evaluación favorable de la agresión sería propia de la agresión proactiva (Roncero, Andreu y Peña, 2016). Según la teoría de Barriga y Gibbs (1996) las distorsiones cognitivas son actitudes, pensamientos y creencias irracionales, que pueden servir para justificar la conducta agresiva, y en tal sentido son distorsiones cognitivas auto-sirvientes (self-serving). 
Estos mecanismos cognitivos sirven al agresor para que proteja su autoestima, minimice el daño, neutralice la empatía y la culpa antes, durante o después del acto agresivo; y están estructurados en dos categorías (Barriga, Hawkins y Camelia, 2008): Distorsiones cognitivas primarias y secundarias; de modo que en la primera categoría el elemento cognitivo primario es el pensamiento egocéntrico, que se caracteriza por el egoísmo y la falta de reflexión en el individuo sobre las consecuencias de sus actos. Por otro lado, las distorsiones cognitivas secundarias cumplen la función de proteger la imagen personal, aliviar el malestar de los actos desviados y liberar de toda responsabilidad por los daños que provocan en otras personas. Estos mecanismos son: Culpar a los demás, en la que el individuo responsabiliza de la agresión a las circunstancias o a los demás, brindando alivio temporal y evitando experimentar sentimientos de humillación, debilidad o inferioridad; Minimización, que supone que al realizar actos agresivos, el individuo tiende a desacreditar a la víctima y cree que actuar agresivamente no es perjudicial o es inofensivo; y Asumir lo peor, que conduce al individuo a atribuir el peor escenario como si fuese inevitable o a percibir una situación como mala de forma permanente (Barriga y Gibbs, 1996).

Por otro lado, así como existen las distorsiones cognitivas auto-sirvientes que permiten justificar las conductas antisociales de los agresores, se ha planteado la existencia de las distorsiones cognitivas auto-humillantes, entre las que se tienen los mecanismos de abstracción selectiva, generalización, catastrofismo y personalización (Peña y Andreu, 2012). La abstracción selectiva supone concentrarse solo en los aspectos negativos de un evento; la generalización como su nombre lo indica, implica considerar que un evento negativo es representativo de otros eventos futuros similares; el catastrofismo supone tener expectativas que se ubican en el peor escenario posible; y la personalización implica atribuir los eventos negativos a causas relacionadas con la propia persona (Ara, 2016).

En ese sentido, diversos estudios han reportado que las distorsiones cognitivas auto-sirvientes se relacionan con conductas agresivas y antisociales, denominadas también externalizantes (Barriga, Landau, Stinson, Liau y Gibbs, 2000); mientras que las distorsiones cognitivas auto-humillantes se asocian con conductas internalizantes como la ansiedad y la depresión (Ara, 2016). En el estudio de Barriga, Hawkins y Camelia (2012) por ejemplo, las distorsiones cognitivas auto-humillantes se asociaron de manera significativa con las conductas internalizantes, a diferencia de las distorsiones auto-sirvientes que se asociaron con conductas delictivas. Por otro lado, la distorsión cognitiva auto-sirviente egocéntrica es un predictor significativo de la agresión proactiva o instrumental, mientras que la distorsión auto-sirviente culpar a los demás predice la agresión reactiva (Peña y Andreu, 2012).

En resumen, las distorsiones cognitivas auto-sirvientes neutralizan los sentimientos de culpa de los agresores a través de la justificación de conductas antisociales; lo que favorece la ocurrencia y el mantenimiento de las conductas agresivas y actos delincuenciales, con evidentes implicancias en el ámbito clínico y forense (Roncero, Andreu y Peña, 2016). Las investigaciones sobre agresión y violencia en el Perú han abarcado temas como la violencia contra la mujer y la violencia familiar (Miljánovich et al., 2013), las relaciones entre la familia y la conducta agresiva en adolescentes (Matalinares et al., 2010) y variables vinculadas con los actos violentos en la escuela (Arias y Rivera, 2019). En la ciudad de Arequipa los estudios sobre agresividad o violencia también han comprendido temas como la violencia familiar o contra la mujer (Castro, Cerellino y Rivera, 2017) y la conducta agresiva de los niños y adolescentes en la escuela, así como los factores determinantes de este tipo de conducta (Arias y Rivera, 2019). Sin embargo, no existen estudios en esta ciudad, sobre la conducta agresiva reactiva/proactiva y las distorsiones cognitivas, debido principalmente a que no se cuenta con instrumentos validados. En consecuencia, el objetivo del presente estudio es analizar las propiedades psicométricas del Reactive/Proactive Questionnaire (RPQ) y del How I Think Questionaire (HIT) de modo que se pueda contar con herramientas debidamente validadas para evaluar esta variable, y aportar a las evidencias psicométricas globales de estos instrumentos y sus respectivos modelos teóricos.

En ese sentido, con respecto a la agresión reactiva/ proactiva, Raine et al. (2006) construyó el Cuestionario de Agresión Reactiva/Proactiva (Reactive/Proactive Aggression Questionnaire - $R P Q$ ), que fue aplicado a adolescentes estadounidenses y que cuenta con validez de constructo y validez convergente, así como índices de confiabilidad superiores a .8, obtenidos mediante el método de consistencia interna y la prueba alfa de Cronbach. La versión en español fue validada por Andreu, Peña y Ramírez (2009) en 732 adolescentes madrileños, encontrando un modelo unidimensional y un modelo bifactorial, siendo este último el que presenta mejores indicadores de ajuste según el análisis factorial confirmatorio. Incluso hay una versión de la prueba que evalúa la conducta agresiva de niños en función de la información proporcionada por sus maestros, que cuenta con una estructura de dos factores que explican el $78 \%$ de la varianza y tienen una confiabilidad alta obtenida mediante 
la prueba alfa de Cronbach (Cosi, Vigil-Colet y Canals, 2009).

Con respecto a las distorsiones cognitivas, Barriga y Gibbs (1996) crearon el Cuestionario "Cómo pienso" ("How I Think" Questionnaire - HIT) para evaluar las distorsiones cognitivas auto-sirvientes; para lo cual aplicaron dicho instrumento en una muestra de adolescentes estadounidenses con conductas antisociales que estaban bajo custodia en el Departamento de Servicios Juveniles en el Estado de Ohio. Los resultados arrojaron índices de confiabilidad adecuados obtenidos mediante el método de test-retest, y validez de constructo con adecuados índices de ajuste. La prueba fue validada en adolescentes españoles por Peña, Andreu, Barriga y Gibbs (2014) quienes tomaron una muestra de 1490 sujetos entre $11 \mathrm{y}$ 19 años. Los resultados reportaron una estructura de seis factores con buen ajuste obtenido por medio de análisis factorial confirmatorio y con índices de consistencia interna superiores a .7 para todos los factores.

\section{Método}

\section{Participantes}

La muestra estuvo conformada por 2830 alumnos de secundaria de diversas instituciones educativas de la ciudad de Arequipa (48.9\% mujeres y 51.1\% varones), cuyas edades fluctúan entre los 13 a 19 años $(\mathrm{M}=15.77$; $\mathrm{DT}=0.847)$. Respecto al grado de instrucción, el $49.7 \%$ de evaluados estudiaba en cuarto grado de secundaria y el $50.3 \%$ el quinto grado. La muestra fue seleccionada mediante métodos no probabilísticos. Las escuelas fueron seleccionadas por conveniencia según las facilidades brindadas por las instituciones educativas para la realización de la investigación y los estudiantes fueron seleccionados mediante la técnica de grupos intactos.

\section{Instrumentos}

Cuestionario de Agresión Reactiva/Proactiva (RQP). El Cuestionario fue desarrollado por Raine et al. (2006) y validado para sujetos hispanoparlantes por Andreu et al. (2009). La prueba consta de 23 ítems con tres niveles de respuesta en una escala de Likert (Nunca, A veces y A menudo). La validación peruana ha sido obtenida en adolescentes de Huaraz, reportando validez de contenido mediante la prueba de Aiken, validez de constructo mediante el análisis factorial confirmatorio e índices de confiabilidad óptimos mediante el coeficiente Omega (Abanto, 2018). En cuanto al análisis factorial confirmatorio se obtuvieron los índices de ajuste de dos modelos psicométricos, uno con 18 ítems y otro con 23 ítems, siendo este último el que obtuvo mejores indicadores de ajuste y mayores índices de confiabilidad, tanto para la dimensión de agresión reactiva $(\omega=.73)$ como para la proactiva $(\omega=.77)$.

How I Think (HIT). El Cuestionario fue desarrollado por Barriga y Gibbs (1996) y adaptado al español por Peña et al. (2013). Es una prueba que consta de 54 ítems de respuesta en una escala Likert de 6 puntos que iba de 1 (Muy en desacuerdo) a 6 (muy de acuerdo), en el cual altos puntajes reflejan altos niveles de distorsiones congnitivas. El HIT consta de 39 ítems para valorar las distorsiones cognitivas autosirvientes (sesgo egocéntrico, culpar a los demás, minimización/etiquetado incorrecto, asumir lo peor) y para cuatro categorías de conducta antisocial del Manual diagnóstico y estadístico de los trastornos mentales, cuarta edición (DSM-IV, American Psychiatric Association, 1994), las cuales son: oposición/desafío, agresión física, mentir y robo. De los restantes 15 ítems, 8 ítems sirven como control para las respuestas anómalas que miden la deseabilidad social y 7 ítems actúan como conductas positivas, los cuales comprenden afirmaciones ligadas a la conducta prosocial.

\section{Procedimiento}

Los estudiantes fueron evaluados en las respectivas aulas de sus respectivas instituciones educativas, previa coordinación con las autoridades de las mismas, las autorizaciones pertinentes y los horarios para la evaluación. Fueron evaluados todos los estudiantes que desearon participar de manera voluntaria, luego de que se les informó acerca de los objetivos del estudio. Los participantes firmaron un consentimiento informado y se les garantizó la reserva y confidencialidad de sus datos, que serán usados solo con fines de investigación.

\section{Análisis de datos}

Primeramente se realización los análisis de los estadísticos descriptivos univariados de los ítems de ambos cuestionarios: media, desviación estándar, asimetría, curtosis; además de la normalidad multivariada por medio del coeficiente de Mardia. Luego de ello se procedió a realizar los análisis factoriales confirmatorios (AFC) con la finalidad de comprobar si la estructura factorial del RPQ y el HIT se replicaban en la realidad peruana. Para el caso del RPQ el primer modelo fue constructo unidimensional en la que la agresión fue hipotetizada como una única variable latente (M1), el segundo fue un modelo de dos factores oblicuos (M2), donde agresión reactiva y proactiva se modelaron de forma conjunta 
Tabla 1. Estadísticos de bondad de ajuste de los modelos propuestos para el RPQ

\begin{tabular}{rccccc}
\hline \multicolumn{1}{c}{ Modelo } & $\chi^{2}(\mathrm{gl})$ & CFI & TLI & RMSEA [IC 90\%] & SRMR \\
\hline Modelo 1 (unifactorial) & $2364.1(230)^{* * *}$ & .979 & .977 & $.057[.055, .059]$ & .062 \\
Modelo 2 (bifactorial) & $2074.1(229)^{* * *}$ & .982 & .980 & $.053[.051 ; .056]$ & .058 \\
\hline
\end{tabular}

Nota $: \chi^{2}=$ Chi cuadrado; $g l=$ Grados de libertad; $C F I=$ índice de ajuste comparativo; TLI= índice de Tuker Lewis; RMSEA = Error Cuadrático Medio de Aproximación; IC = Intervalos de confianza; SRMR = raíz residual estandarizada cuadrática media.

${ }^{* * *} p<.001$.

(Raine et al., 2006). En cuanto al HIT el modelo evaluado fue el propuesto por Barriga et al. (2001), el cual refiere que el HIT se compone de un factor que evalúa las distorsiones cognitivas (egocentrismo, culpar a otros, minimizar/etiquetar y asumir lo peor); incluyendo la presencia de dos factores adicionales, respuestas anómalas y conductas positivas. Cabe resaltar que no se analizaran las 4 factores conductuales que evalúa el HIT dado que este estudio se centra en las distorsiones cognitivas $\mathrm{y}$ al hecho de que los ítems del HIT, por como la prueba fue construida (Barriga et al. 2008), pueden saturar tanto en alguna de las distorsiones cognitivas como en los factores conductuales y ello podría generar confusión.

Para realizar los análisis estadísticos se utilizó el software R versión 3.5.2 (R Core Team, 2018), concretamente para realizar los análisis descriptivos univariados y variados se usó el paquete y psych versión 1.8.12 (Revelle, 2018) y para el AFC se utilizó el paquete lavaan versión 0.5-23.1097 (Rosseel, 2012). Además, estimamos la fiabilidad de los test por medio de los coeficientes $\alpha$ de Cronbach y $\omega$ de McDonald. Finalmente, luego de validados los instrumentos, se procedió a correlacionar las dimensiones de los mismos por medio del coeficiente Rho se Spearman, teniendo en cuenta que las dimensiones del RPQ no presentaron una distribución normal, para ello utilizamos el software JASP versión 0.9.2 (JASP Team, 2018).

\section{Resultados}

\section{Análisis psicométrico del $R P Q$}

El análisis descriptivo univariado de los ítems del RPQ, evidenciaron que la asimetría y el exceso de curtosis estuvieron mayormente por fuera del intervalo $[-1 ; 1]$ en 12 de los 23 ítems que componen el cuestionario (ítem 1, 9, 10, 12, 15, 16, 17, 18, 20, 21, 22 y 23), lo que aunado a un coeficiente de normalidad multivariada de Mardia (137.15) que fue mayor a 70, nos indica que los ítems no siguen una distribución normal (Muthén y Kaplan, 1985). Es por ello que se decidió por el método de estimación de mínimos cuadrados diagonalmente ponderados robustos (DWLS), el cual fue propuesto por
Muthen (1993) para el análisis de ítems categóricos sobre la base de correlaciones policóricas. Dicho método de estimación ha mostrado ser eficaz en muestras pequeñas y con distribuciones no normales (Rhemtulla, Brosseau-Laird y Savalei, 2012).

En la Tabla 1 se puede observar el contraste de dos modelos estructurales del RPQ, uno unifactorial y otro bifactorial, ambos modelos muestran buenos índices de bondad de ajuste; sin embargo, el modelo bifactorial (agresión reactiva y agresión proactiva) es el que mejor se acerca a la realidad.

En la Tabla 2 se pueden ver las cargas factoriales de cada uno de los ítems en sus respectivos factores, los cuales fluctúan entre .491 a .814; además cabe resaltar que no se eliminó ningún ítem durante el AFC.

Tabla 2. Factores y cargas factoriales del RPQ

\begin{tabular}{lccc}
\hline Reactiva & Carga factorial & Proactiva & Carga factorial \\
\hline Ítem 1 & .580 & Ítem 2 & .703 \\
Ítem 3 & .685 & Ítem 4 & .491 \\
Ítem 5 & .518 & Ítem 6 & .587 \\
Ítem 7 & .604 & Ítem 9 & .774 \\
Ítem 8 & .649 & Ítem 10 & .742 \\
Ítem 11 & .676 & Ítem 12 & .772 \\
Ítem 13 & .579 & Ítem 15 & .788 \\
Ítem 14 & .567 & Ítem 17 & .783 \\
Ítem 16 & .769 & Ítem 18 & .633 \\
Ítem 19 & .656 & Ítem 20 & .675 \\
Ítem 22 & .771 & Ítem 21 & .797 \\
& & ítem 23 & .814 \\
\hline
\end{tabular}

\section{Análisis psicométrico del HIT}

Respecto a los descriptivos univariados de los ítems del HIT, se encontró que la asimetría y el exceso de curtosis estuvieron mayormente por fuera del intervalo $[-1 ; 1]$ en 27 de los 54 ítems del cuestionario (ítem 5, 7, $9,11,12,13,16,17,19,23,24,27,28,30,34,35,38$, $39,40,41,42,43,45,47,48,53$ y 54$)$, lo que aunado 
a un coeficiente de normalidad multivariada de Mar$\mathrm{dia}=242.15$ y que es mayor a 70 , nos indica que los ítems no siguen una distribución normal (Muthén y Kaplan, 1985). Teniendo en cuenta lo anteriormente mencionado, no es recomendable usar el método de máxima verosimilitud (Pérez, \& Medrano, 2010), por lo que se optó por usar el método de estimación de mínimos cuadrados diagonalmente ponderados robustos (DWLS).

En la Tabla 3 se puede observar que el AFC confirmó la estructura factorial propuesta por Barriga, Gibbs, Potter y Liau (2001), la cual refiere que el HIT se compone de 4 factores que miden las distorsiones cognitivas (egocentrismo, culpar a otros, minimizar/etiquetar y asumir lo peor); así como, la presencia de dos factores adicionales, respuestas anómalas y conductas positivas. Los índices de ajuste fueron mayormente adecuados para validar los modelos, con un CFI y TLI mayores de .9 y RMSEA y SRMR menores de .08; con la excepción de la chi-cuadrada de bondad de ajuste $\left(\chi^{2}=15968.2 ; \mathrm{p}<.001\right)$ que obtuvo un p-valor menor de .05 , lo cual es esperado en muestras grandes.

En la Tabla 4 se pueden ver las cargas factoriales de cada uno de los ítems en sus respectivos factores, los cuales fluctúan entre .308 a .767. Por otro lado, cabe mencionar que los ítems 1, 2, 4, 6 y 19 se tuvieron que eliminar por presentar cargas factoriales menores a 3 .

\section{Análisis correlacional entre el HIT y el RPQ}

En la Tabla 5, se presentan los estadísticos descriptivos de las dimensiones tanto del HIT como del RPQ, evidenciando que la asimetría y el exceso de curtosis de todas las dimensiones del HIT estuvieron dentro del intervalo $[-1 ; 1]$; por el contrario, las dos dimensiones del RPQ tienen una asimetría y el exceso de curtosis fuera del intervalo $[-1 ; 1]$. Teniendo en cuenta este hecho lo recomendable es procesar nuestros datos con el coeficiente de correlación Rho de Spearman. Además se presentan los coeficientes de confiabilidad, siendo así que las dimensiones del RPQ, agresión reactiva $(\alpha=.826 ; \omega=$ .828) y agresión proactiva $(\alpha=.852 ; \omega=.863)$ presentan adecuados indicadores de confiabilidad. De la misma manera las diferentes dimensiones del HIT presentaron coeficientes de confiabilidad adecuados ya que fluctuaron entre $\alpha=.714$ a .834 y $\omega=.721$ a .840 .

En la Tabla 6 apreciamos que la agresión reactiva se relaciona directa y significativamente con todas las distorsiones cognitivas y conductuales evaluadas por el HIT, siendo la mayoría de coeficientes de una intensidad débil. Asimismo, la agresión proactiva también se relaciona directa y significativamente con todas las distorsiones cognitivas y conductuales evaluadas por el HIT; aunque en este caso la mayoría de coeficientes tienen una intensidad moderada.

\section{Discusión}

En el presente estudio se pretendió analizar las propiedades psicométricas del Reactive/Proactive Questionnaire (Raine et al., 2009) y del cuestionario How I Think (Barriga y Gibbs, 1996) en una muestra adolescentes de la ciudad de Arequipa, en Perú. Cabe señalar que ambos instrumentos, han sido analizados psicométricamente en diversas ocasiones, demostrando tener adecuados índices de validez y confiabilidad. En ese sentido, nuestros resultados también han encontrado que el RPQ tiene dos factores que diferencian entre la agresión reactiva y proactiva, con índices de consistencia interna superiores a .8 para sus dos factores, tanto con la prueba Alfa de Cronbach como con la prueba Omega de McDonald; datos que son similares a los reportados en los estudios de Andreu (2009, 2010; Andreu et al., 2009) en adolescentes hispanoparlantes, y de Abanto (2018) con adolescentes peruanos de la provincia de Huaraz, al norte de Perú. Por otro lado, el HIT ha demostrado tener un ajuste adecuado tanto para sus factores cognitivos como conductuales, con índices de consistencia interna superiores a .7, mediante la prueba Alfa de Cronbach y Omega de McDonald, para cada uno de los factores cognitivos y conductuales; tal y como ha sido reportado en estudios previos de corte psicométrico (Barriga y Gibbs, 1996; Barriga, Gibbs, Potter y Liau, 2001; Peña, Andreu, Barriga y Gibbs, 2014).

Ambos instrumentos han resultado ser válidos y confiables, lo cual se ratifica también con los datos correlaciónales obtenidos, que prueban que los constructos que evalúan dichos instrumentos se encuentran relacionados.

Tabla 3. Estadísticos de bondad de ajuste de los factores cognitivos y conductuales del HIT

\begin{tabular}{cccccc}
\hline Modelo & $\chi^{2}(\mathrm{gl})$ & CFI & TLI & RMSEA [IC 90\%] & SRMR \\
\hline Modelo 1 & $15968.2(1112)^{* * *}$ & .957 & .955 & $.069[.068, .070]$ & .068 \\
\hline
\end{tabular}

Nota: $\chi^{2}=$ Chi cuadrado; $g l=$ Grados de libertad; CFI= índice de ajuste comparativo; TLI= índice de Tuker Lewis; RMSEA = Error Cuadrático Medio de Aproximación; IC = Intervalos de confianza; SRMR = raíz residual estandarizada cuadrática media.

${ }^{* * * *} p<.001$. 
Tabla 4. Factores y cargas factoriales del HIT

\begin{tabular}{|c|c|c|c|c|c|c|}
\hline & Egocentrismo & Minimizar/etiquetar & Respuestas anómalas & Culpar a otros & Asumir lo peor & Conductas positivas \\
\hline Ítem 3 & .514 & & & & & \\
\hline Ítem 7 & .575 & & & & & \\
\hline Ítem 10 & .548 & & & & & \\
\hline Ítem 22 & .622 & & & & & \\
\hline Ítem 28 & .752 & & & & & \\
\hline Ítem 37 & .594 & & & & & \\
\hline Ítem 42 & .323 & & & & & \\
\hline Ítem 52 & .540 & & & & & \\
\hline Ítem 54 & .713 & & & & & \\
\hline Ítem 5 & & .554 & & & & \\
\hline Ítem 12 & & .640 & & & & \\
\hline Ítem 14 & & .582 & & & & \\
\hline Ítem 17 & & .591 & & & & \\
\hline Ítem 30 & & .704 & & & & \\
\hline Ítem 33 & & .605 & & & & \\
\hline Ítem 40 & & .630 & & & & \\
\hline Ítem 47 & & .674 & & & & \\
\hline Ítem 13 & & & .680 & & & \\
\hline Ítem 20 & & & .512 & & & \\
\hline Ítem 27 & & & .720 & & & \\
\hline Ítem 31 & & & .493 & & & \\
\hline Ítem 38 & & & .560 & & & \\
\hline Ítem 45 & & & 619 & & & \\
\hline Ítem 51 & & & .590 & & & \\
\hline Ítem 11 & & & & .553 & & \\
\hline Ítem 21 & & & & .689 & & \\
\hline Ítem 25 & & & & .472 & & \\
\hline Ítem 26 & & & & .553 & & \\
\hline Ítem 36 & & & & .532 & & \\
\hline Ítem 39 & & & & .681 & & \\
\hline Ítem 44 & & & & .708 & & \\
\hline Ítem 46 & & & & .577 & & \\
\hline Ítem 50 & & & & .724 & & \\
\hline Ítem 8 & & & & & .308 & \\
\hline Ítem 15 & & & & & .434 & \\
\hline Ítem 18 & & & & & .520 & \\
\hline Ítem 23 & & & & & .376 & \\
\hline Ítem 29 & & & & & .467 & \\
\hline Ítem 32 & & & & & .653 & \\
\hline Ítem 35 & & & & & .664 & \\
\hline Ítem 43 & & & & & .724 & \\
\hline Ítem 49 & & & & & .527 & \\
\hline Ítem 53 & & & & & .742 & \\
\hline Ítem 9 & & & & & & .461 \\
\hline Ítem 16 & & & & & & .724 \\
\hline Ítem 24 & & & & & & .738 \\
\hline Ítem 34 & & & & & & .741 \\
\hline Ítem 41 & & & & & & .714 \\
\hline Ítem 48 & & & & & & .596 \\
\hline
\end{tabular}


Tabla 5. Estadísticos descriptivos de las escalas del HIT y del RPQ

\begin{tabular}{llrrrrrr}
\hline \multicolumn{1}{c}{$n=2830$} & $M$ & DT & $g 1$ & $g 2$ & $\alpha$ & $\omega$ \\
\hline RPQ & Agresión reactiva & 7.83 & 4.023 & 1.042 & 2.324 & .826 & .828 \\
& Agresión proactiva & 3.70 & 3.62 & 1.712 & 4.196 & .852 & .863 \\
HIT & Egocentrismo & 20.19 & 6.23 & 0.523 & 0.366 & .766 & .772 \\
& Culpar a otros & 19.06 & 6.218 & 0.499 & 0.067 & .788 & .791 \\
& Minimizar/etiquetar & 15.58 & 5.366 & 0.685 & 0.258 & .763 & .765 \\
& Asumir lo peor & 23.03 & 6.227 & 0.314 & 0.016 & .733 & .742 \\
& Respuestas anómalas & 21.50 & 5.727 & .092 & -0.357 & .749 & .749 \\
& Conductas positivas & 28.09 & 5.001 & -1.039 & 0.988 & .754 & .755 \\
\hline
\end{tabular}

Nota: $M=$ media, DT= desviación típica, $g 1=$ asimetría, $g 2=$ curtosis, $\alpha=$ Alpha de Cronbach, $\omega=$ Omega de McDonald.

Tabla 6. Correlación entre las dimensiones del HIT y del RPQ

\begin{tabular}{|c|c|c|c|c|c|c|c|c|}
\hline & 1 & 2 & 3 & 4 & 5 & 6 & 7 & 8 \\
\hline 1. Agresión reactiva & - & & & & & & & \\
\hline 2. Agresión proactiva & $.665^{* * *}$ & - & & & & & & \\
\hline 3. Egocentrismo & $.411^{* * *}$ & $.468^{* * *}$ & - & & & & & \\
\hline 4. Culpar a otros & $.375^{* * *}$ & $.405^{* * *}$ & $.736^{* * *}$ & - & & & & \\
\hline 5. Minimizar/etiquetar & $.314^{* * *}$ & $.400^{* * *}$ & $.716^{* * *}$ & $.744^{* * *}$ & - & & & \\
\hline 6. Asumir lo peor & $.380^{* * *}$ & $.411^{* * *}$ & $.696^{* * *}$ & $.752^{* * *}$ & $.708^{* * *}$ & - & & \\
\hline 7. Respuestas anómalas & $.431^{* * *}$ & $.455^{* * *}$ & $.572^{* * *}$ & $.526^{* * *}$ & $.460^{* * *}$ & $.526^{* * *}$ & - & \\
\hline 8. Conductas positivas & $-.047^{*}$ & $-.144^{* * *}$ & $-.231^{* * *}$ & $-.304^{* * *}$ & $-.396^{* * *}$ & $-.265^{* * *}$ & .037 & - \\
\hline
\end{tabular}

${ }^{* * *} p<.001 ;{ }^{*} p<.05$.

En ese sentido, se ha encontrado que, tal y como se ha reportado en otros estudios previos, las distorsiones cognitivas se correlacionan positiva y significativamente con la conducta agresiva reactiva y proactiva (Barriga, Hawkins y Camelia, 2008; Barriga, Landau, Stinson, Liau y Gibbs, 2000). Con la salvedad de que la agresión proactiva se correlaciona de manera más fuerte con las distorsiones cognitivas, que la agresión reactiva, esto no solo ha sido corroborado por otros estudios (Roncero, Andreu y Peña, 2016), sino que tiene sustento en el hecho de que la agresión proactiva es un tipo de conducta agresiva deliberada, planificada; que supone ciertos esquemas cognitivos fijados, que a su vez reflejan una manera de ver y entender el mundo circundante; mientras que la agresión reactiva responde más al contexto de la expresión de ira en un momento dado.

En ese sentido, los esquemas cognitivos tienen un peso determinante en diversos contextos de violencia, tanto entre los agresores como en las víctimas. Así pues, tenemos que las mujeres maltratadas presentan esquemas disfuncionales que justifican las agresiones ejercidas por sus parejas (Huerta, Ramírez, Ramos, Murillo,
Falcón, Misare y Sánchez, 2016), mientras los agresores o personas con conductas abiertamente antisociales por otro lado, también presentan esquemas cognitivos distorsionados (Barriga, Hawkins y Camelia, 2008).

Por ello, del presente trabajo se desprenden algunas implicaciones prácticas que deseamos comentar. En primer lugar, dado que los resultados del procesamiento psicométrico aplicado al Cuestionario de Agresión Reactiva Proactiva y al Cuestionario "How I think", indican que son válidas y confiables, se encuentra sustento psicométrico a su uso en adolescentes de la ciudad de Arequipa, lo cual permitirá la realización de investigaciones sobre las variables de conducta agresiva reactiva proactiva y distorsiones cognitivas. En segundo lugar, se encontró evidencia de que las variables de conducta agresiva reactiva proactiva y distorsiones cognitivas se encuentran correlacionadas, dando sustento teórico a los estudios que confirman estas relaciones. En tercer lugar, la validación de estos instrumentos favorecerán su uso en los ámbitos clínicos, educativos y jurídicos, donde se suele evaluar la conducta agresiva de los adolescentes, y en relación a ello, las distorsiones cognitivas. 
Ahora bien, entre las principales limitaciones de este estudio, se tiene el tipo de muestreo que no ha seguido métodos probabilísticos, sin embargo, el tamaño de la muestra es representativo de la población de estudio. Por otro lado, no existen reportes previos sobre estas variables de estudio en el Perú, por lo que un contraste con estudios similares en nuestro medio, ha sido imposible; pero se han empleado estudios similares realizados en Estados Unidos y Europa, y otros realizados localmente sobre variables relacionadas.

Al margen de estas limitaciones, el presente trabajo hace un aporte teórico y metodológico, pues no solo ofrece las propiedades psicométricas de dos instrumentos que no han sido empleados en nuestro medio local, sino que también valora dos constructos psicológicos importantes hoy en día. Posiblemente la presente investigación, dé inicio a otros estudios sobre la agresión reactiva/proactiva y las distorsiones cognitivas, en relación a otras variables de riesgo en los adolescentes, tales como consumo de sustancias psicoactivas, factores familiares y socioculturales asociados. Asimismo, se sugiere abordar estas temáticas mediante diferentes diseños metodológicos, tales como estudios descriptivos, comparativos, correlacionales, causales, etc.

\section{Referencias}

Abanto, A. L. (2018). Evidencias de validez del cuestionario de agresión reactiva y proactiva ( $R P Q)$ en adolescentes de Huaraz. (Tesis de pregrado). Escuela Profesional de Psicología. Universidad César Vallejo, Trujillo, Perú.

Amor, P. J., Echeburúa, E., y Loinaz, I. (2009). ¿Se puede establecer una clasificación tipológica de los hombres violentos contra su pareja? International Journal of Clinical and Health Psychology, 9(3), 519-539.

Andreu, J. M. (2009). Propuesta de un modelo integrador de la agresividad impulsiva y premeditada en función de sus bases motivacionales y socio-cognitivas. Psicopatología Clínica Legal y Forense, 9, 85-98.

Andreu, J. M. (2010). Cuestionario de Agresividad Impulsiva y Premeditada en Adolescentes (CAPI-A). Madrid: TEA Ediciones.

Andreu, J. M., y Peña, E. (2013). Escala de conducta antisocial y delictiva. Anales de Psicología, 29, 516-522.

Andreu, J. M., Peña, E., y Ramírez, J. M. (2009). Cuestionario de agresión reactiva y proactiva: Un instrumento de medida de la agresión en adolescentes. Revista de Psicopatología y Psicología Clínica, 14(1), 37-49.

Andreu, J. M., Peña, E., y Penado, M. (2012). Análisis de la impulsividad en diferentes grupos de adolescentes agresivos. International Journal of Psychology and Psychological Therapy, 12, 441-452.

Andreu, J. M., Peña, E., y Penado, M. (2013). Impulsividad cognitiva, conductual y no planificadora en adolescentes agresivos reactivos, proactivos y mixtos. Anales de Psicología, $3(2), 730-740$.

Ara, E. (2016). Measuring self-debasing cognitive distortions in youth. International Journal of Asian Social Science, 6(12), 705-712.

Arias, W. L., y Rivera, R. (2019). Acoso escolar: análisis teórico e investigación sobre el bullying en el Perú. En J. C. Carozzo \& J. Chahuara (Comps.), Bullying, convivencia y algo más (pp. 57-97). Lima: Universidad Santo Domingo de Guzmán.

Barriga, A. Q., y Gibbs, J. C. (1996). Measuring cognitive distortion in antisocial youth: Development and preliminary validation of the "How I think" Questionnaire. Aggressive Behavior, 22, 333-343.

Barriga, A. Q., Gibbs, J. C., Potter, G., y Liau, A. K. (2001). The How I Think Questionnaire Manual. Champaign, IL: Research Press.

Barriga, A. Q., Hawkins, M. A., y Camelia, C. R. T. (2008). Specificity of cognitive distortions to antisocial behaviours. Criminal Behavior and Mental Health, 18, 104-116.

Barriga, A. Q., Landau, J. R., Stinson, B. L., Liau, A. K., y Gibbs, J. C. (2000). Cognitive distortion and problem behaviors in adolescence. Criminal Justice and Behavior, 27, 36-56.

Bandura, A. (1965). Influence of models' reinforcement contingencies on the acquisition of imitative responses. Journal of Personality and Social Psychology, 1(6), 589-595.

Boira, S., y Jodrá, P. (2010). Psicopatología, características de la violencia y abandonos en programas para hombres violentos con la pareja: resultados en un dispositivo de intervención. Psicothema, 22(4), 593-599.

Castro, R., Cerellino, L. P., y Rivera, R. (2017). Risk factors of violence against women in Peru. Journal of Family Violence, 32(8), 807-815. doi: 10.1007/s10896-017-9929-0

Cosi, S., Vigil-Colet, A., y Canals, J. (2009). Desarrollo del cuestionario de agresividad proactiva/reactiva para profesores: estructura factorial y propiedades psicométricas. Psicothema, 21(1), 159-164.

Dodge, K., y Coie, J. D. (1987). Social information processing factors in reactive and proactive aggression in children's peer group. Journal of Personality and Social Psychology, 53, 1146-1158.

Dodge, K., Lochman, J. E., Harnish, J. D., y Bates, J. E. (1997). Reactive and proactive aggressive in school children and psychiatrically impaired chronically assaultive youth. Journal of Abnormal Psychology, 106, 37-51.

Dollard, J., Doob, L. W., Miller, N. E., Mowrer, O. H., y Sears, R. R. (1939). Frustration and aggression. New Heaven: Yale University Press.

Fares, N., Cabrera, J. M., Lozano, F., Salas, F., y Ramírez, J. M. (2012). Agresión reactiva y proactiva en niños y adolescentes uruguayos. Psicopatología Clínica Legal y Forense, 12, 4768.

Fernández, J., y Echeburúa, E. (2008). Trastornos de personalidad y psicopatía en hombres condenados por violencia grave contra la pareja. Psicothema, 20(2), 193-198.

Gallardo-Pujol, D., Forero, C. G., Maydeu-Olivares, A., y AndrésPueyo, A. (2009). Desarrollo del comportamiento antisocial. Factores psicobiológicos, ambientales e interacciones gentipoambiente. Revista de Neurología, 48(4), 191-198. 
Garaigordobil, M., Durá, A., y Pérez, J. I. (2005). Síntomas psicopatológicos, problemas de conducta y autoconceptoautoestima: Un estudio con adolescentes de 14 a 17 años. Anuario de Psicología Clínica y de la Salud, 1, 53-63.

Herrera, D., y Morales, H. (2005). Comportamiento antisocial durante la adolescencia: Teoría, investigación y programas de prevención. Revista de Psicología (Pontificia Universidad Católica del Perú), 23(2), 201-247.

Huerta, R., Ramírez, N., Ramos, J., Murillo, L., Falcón, C., Misare, M., y Sánchez, J. (2016). Esquemas cognitivos disfuncionales y dependencia emocional en mujeres con y sin violencia en la relación de pareja de la ciudad de Lima. Revista de Investigación en Psicología, 19(2), 145-192

JASP Team (2018). JASP (Version 0.9.2) [Computer software].

Latinobarómetro (2018). Informe 2018. Santiago, Chile: Corporación Latinobarómetro.

Matalinares, M., Arenas, C., Sotelo, L., Díaz, G., Dioses, A., Yaringaño, J., Muratta, R., Pareja, C., y Tipacti, R. (2010). Clima familiar y agresividad en estudiantes de secundaria de Lima Metropolitana. Revista de Investigación en Psicología, 13(1), 109-128.

Miljánovich, M. A., Huerta, R. E., Campos, E., Torres, S., Vásquez, V. A., Vera, K., y Díaz, A. (2013). Violencia familiar: modelos explicativos del proceso a través del estudio de casos. Revista de Investigación en Psicología, 16(1), 29-44.

Muthén, B. O. (1993). Goodness of fit with categorical and other nonnormal variables. In K.A. Bollen, \& J.S. Long (Eds.). Testing structural equation Models (pp. 205-234). Newbury Park, CA: Sage.

Muthén, B., y Kaplan D. (1985). A comparison of some methodologies for the factor analysis of non-normal Likert variables. British Journal of Mathematical and Statistical Psychology, 38, 171-189.

Penado, M., Andreu, J. M., y Peña, E. (2014). Agresividad reactiva, proactiva y mixta: análisis de los factores de riesgo individual. Anuario de Psicología Jurídica, 24, 37-42.

Peña, M. E., y Andreu, J. M. (2012). Distorsiones cognitivas: Una revisión sobre sus implicaciones en la conducta agresiva y antisocial. Psicopatología Clínica, Legal y Forense, 12, 8599.
Peña, E., Andreu, J. M., Barriga, A., y Gibbs, J. (2014). Psychometrical properties of the "How I think" Questionnaire (HIT-Q) in adolescents. Psicothema, 25(4), 542-548.

Pérez, E. R., y Medrano, L. A. (2010). Análisis factorial exploratorio: bases conceptuales y metodológicas. Revista Argentina de Ciencias del Comportamiento, 2(1), 58-66.

Quiroz, N., Villatoro, J. A., Juárez, F., Gutiérrez, M. L., Amador, N. G., y Medina-Mora, M. E. (2007). La familia y el maltrato como factores de riesgo de conducta antisocial. Salud Mental, 30(4), 47-54.

Raine, A., Dodge, K., Loeber, R., Gatzke-Kopp, L., Lynam, D., Reynolds, C., Stouthamer-Loeber, M., y Liu, J. (2006). The Reactive-Proactive Aggression Questionnaire: Differential correlates of reactive and proactive aggression in adolescent boys. Aggressive Behavior, 32, 159-171.

R Core Team (2018). R: A language and environment for statistical computing. R Foundation for Statistical Computing, Vienna, Austria. URL https://www.R-project.org/.

Revelle, W. (2018). Psych: Procedures for Personality and Psychological Research, Northwestern University, Evanston, Illinois, USA. URL: https://CRAN.R-project.org/ package $=$ psych Version $=1.8 .12$.

Rhemtulla, M., Brosseau-Liard, P. É., y Savalei, V. (2012). When can categorical variables be treated as continuous? A comparison of robust continuous and categorical SEM estimation methods under suboptimal conditions. Psychological Methods, 17, 354-373.

Rodríguez, A. (2003). Interacción familiar y conducta antisocial. Boletín de Psicología, 78, 7-19.

Roncero, D., Andreu, J. M., y Peña, M. E. (2016). Procesos cognitivos distorsionados en la conducta agresiva y antisocial en adolescentes. Anuario de Psicología Jurídica, 26, 88-101.

Rosseel, Y. (2012). lavaan: An R Package for Structural Equation Modeling. Journal of Statistical Software, 48(2), 1-36.

Torregrosa, M. S., Inglés, C., García-Fernández, J. M., RuízEsteban, C., López-García, K. S., y Zhou, X. (2010). Diferencias en la conducta agresiva entre adolescentes españoles, chinos y mexicanos. European Journal of Education and Psychology, 3(2), 167-176. 